\title{
Something to SNF about
}

\author{
Mark A Gillespie \& Michael A Rudnicki \\ Factors that modify chromatin are crucial for regulating gene expression, but what, in turn, regulates these factors? A \\ new study highlights the importance of signaling cascades in recruiting chromatin-remodeling enzymes to specific \\ promoters during muscle differentiation.
}

Skeletal muscle differentiation relies on signals that induce the transcriptional activity of MyoD, a basic helix-loop-helix transcription factor that acts as a key regulator of the muscle determination program. Muscle differentiation also depends on chromatin-modifying factors that acetylate and remodel histones to unravel chromatin and allow further access of transcriptional machinery to promoters ${ }^{1}$. The differentiation-activated $\mathrm{p} 38 \alpha / \beta$ signaling pathway also stimulates the transcriptional activity of $\mathrm{MyoD}^{2}$, but it was not clear whether this occurred directly, through MyoD phosphorylation, or indirectly, perhaps through chromatin modifiers. On page 738 , Simone and colleagues ${ }^{3}$ show that p38 $\alpha / \beta$ signals directly to the SWI-SNF chromatin remodeling complex, thereby recruiting SWI-SNF to specific target promoters, where it cooperates with MyoD to activate transcription of muscle-specific genes.

\section{Modifying chromatin}

Previous work showed that inhibiting the p38 $\alpha / \beta$ pathway with the drug SB203580 prevents myoblast differentiation due to the inability of MyoD to activate transcription $^{2,4,5}$. To further clarify the mechanism and test the hypothesis that $\mathrm{p} 38 \alpha / \beta$ indirectly regulates MyoD activity, Simone et al. examined the effects of SB203580 on chromatin modifications at the promoters of the differentiation genes myogenin (Myog) and muscle creatine kinase $(\mathrm{Ckm})$. They found that the acetylation status of histones $\mathrm{H} 3$ and $\mathrm{H} 4$ was unaltered by inhibition of $\mathrm{p} 38 \alpha / \beta$ signaling. Consistent with this, the interaction between MyoD and the histone acetyltransferases p300 and PCAF $^{6}$ still occurred at these promoters in the absence of $\mathrm{p} 38 \alpha / \beta$ signaling. These results suggest that, even when functional MyoDacetyltransferase complexes are present on these promoters, activation of gene expression requires a further signal from $\mathrm{p} 38 \alpha / \beta$.

MyoD contains two domains that mediate

Mark A. Gillespie and Michael A. Rudnicki are at the Ottawa Health Research Institute, Molecular Medicine Program, Ottawa, Ontario K1H 8L6, Canada.

e-mail:mrudnicki@ohri.ca the remodeling of chromatin ${ }^{7}$, and SWI-SNF chromatin remodeling activity is also necessary for MyoD-mediated differentiation ${ }^{8}$. Simone et al. therefore tested whether $\mathrm{p} 38 \alpha / \beta$ signaling affects chromatin remodeling at the Myog and $\mathrm{Ckm}$ promoters. Indeed, they found that chromatin was not remodeled in the absence of $p 38 \alpha / \beta$ signaling, suggesting several possibilities: (i) $\mathrm{p} 38 \alpha / \beta$ signals directly to $\mathrm{MyoD}$ to activate its intrinsic chromatin remodeling activity; (ii) $\mathrm{p} 38 \alpha / \beta$ signaling recruits the SWI-SNF complex to promoters; (iii) $\mathrm{p} 38 \alpha / \beta$ activates the SWISNF complex already present on promoters; or (iv) some combination of the above.

\section{Targeting SWI-SNF}

To narrow down the possibilities, Simone et al. examined the association of BRG1 and BRM, the ATPase subunits of the SWI-SNF complex, with the Myog and $\mathrm{Ckm}$ promoters. They found that inhibition of $\mathrm{p} 38 \alpha / \beta$ prevented the association of BRG1 and BRM with $\mathrm{MyoD}$, implying that $\mathrm{p} 38 \alpha / \beta$ signaling is involved in recruiting the SWI-SNF complex to specific myogenic promoters. They also showed that $\mathrm{p} 38 \alpha / \beta$ phosphorylates the BAF60 subunit of the SWI-SNF complex in vitro, suggesting a direct link between $\mathrm{p} 38 \alpha / \beta$ and SWI-SNF activity.

These studies show that blocking $\mathrm{p} 38 \alpha / \beta$ activity results in a failure to recruit the SWI-SNF complex to myogenic promoters. What happens when myoblasts are forced to differentiate by the overexpression of the upstream $\mathrm{p} 38 \alpha / \beta$ activator MKK6? Simone et al. found that forced activation of the p38 $\alpha / \beta$ pathway in myoblasts resulted in premature association of MyoD with BRG1, p300 and PCAF on the Myog promoter, with only the MyoD-BRG1 association dependent on $\mathrm{p} 38 \alpha / \beta$ signaling.

To determine whether $\mathrm{p} 38 \alpha / \beta$ signals directly to MyoD in addition to the SWI-SNF complex, Simone et al. transfected SW13 cells deficient in BRG1 and BRM with constructs expressing Gal4 fused to full-length MyoD or to a truncated form of MyoD lacking the chromatin-remodeling domains. They found that neither Gal4 fusion protein

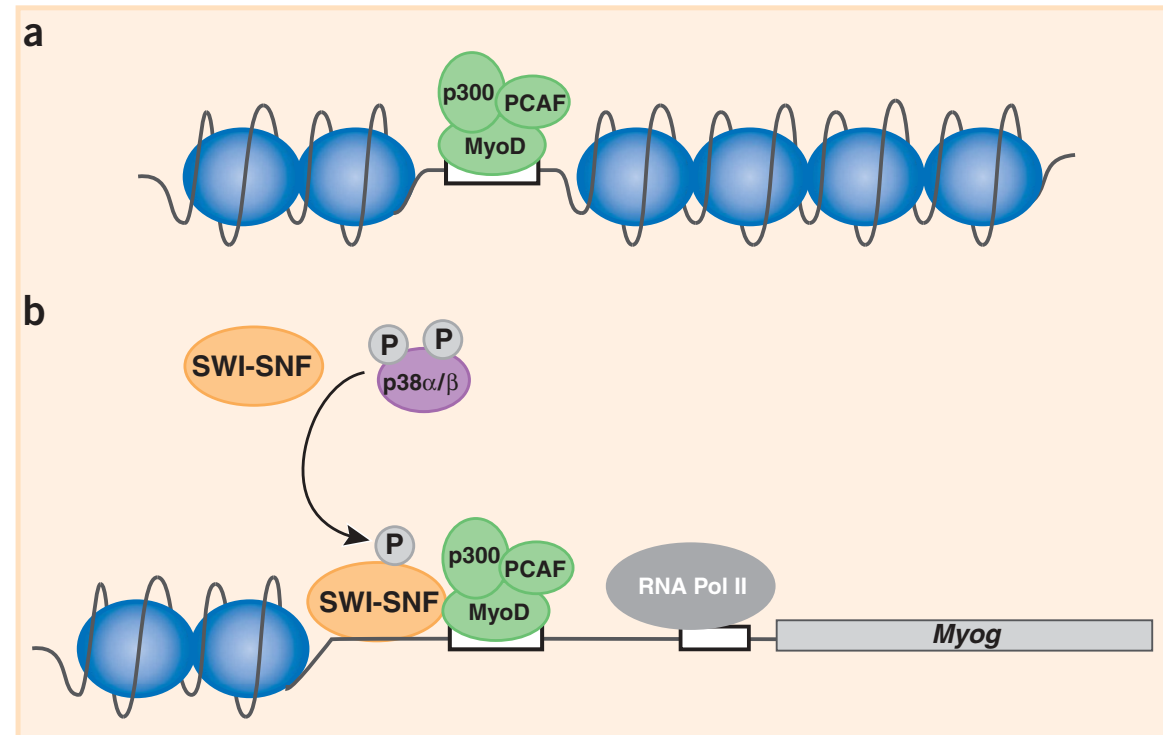

Figure 1 Regulation of MyoD-dependent gene expression by $p 38 \alpha / \beta$. (a) The MyoD-p300-PCAF complex is bound to DNA but unable to activate transcription in the absence of further signals. (b) $p 38 \alpha / \beta$ targets SWI-SNF to the MyoD-p300-PCAF complex, resulting in remodeling of chromatin at the Myog and $\mathrm{Ckm}$ promoters and activation of gene expression. 
could activate a Gal4-dependent reporter further in response to MKK6-induced p38 $\alpha / \beta$ signaling. In fact, further activation of the reporter was not observed until either BRG1 or BRM was added back to these cells. These results provide compelling evidence that $\mathrm{p} 38 \alpha / \beta$ does not signal directly to MyoD or affect its intrinsic remodeling activity.

Mef2 proteins cooperate with MyoD to induce gene expression ${ }^{9}$ and are also targets of p38 $\alpha / \beta$ signaling ${ }^{2,5,10}$. Could Mef2 proteins be mediating some of the observed effects? Notably, the truncated version of MyoD, which lacks the ability to associate with Mef2 (ref. 11), retains the ability to activate the reporter in the presence of BRG1 or BRM and $\mathrm{p} 38 \alpha / \beta$ signaling. These findings argue against an essential role for Mef2 under the assay conditions used, but further studies are required to examine this question in detail.

\section{Pathways to activation}

This study by Simone et al. provides evidence that the $\mathrm{p} 38 \alpha / \beta$ pathway promotes muscle differentiation by indirectly signaling to MyoD through the SWI-SNF chromatinremodeling complex, resulting in expression of $\mathrm{Myog}$ and $\mathrm{Ckm}$ (Fig. 1). Increasing evidence over the past few years suggests that, in addition to targeting transcription factors, kinases can alter gene expression by targeting chromatin-modification factors. Simone et al. have demonstrated this for the first time with the $\mathrm{p} 38 \alpha / \beta$ pathway in muscle.

As $\mathrm{p} 38 \alpha / \beta$ regulates only a small proportion of MyoD target genes ${ }^{12}$, it will be interesting to determine if other promyogenic signaling molecules are responsible for recruiting chromatin modifiers to different loci. It will also be important to determine exactly how BAF60 phosphorylation influences the selective recruitment of SWI-SNF to specific target loci. Are all p38 $\alpha / \beta$ regulated targets in muscle activated by this mechanism, or do Mef2 proteins mediate some of the observed effects of $\mathrm{p} 38 \alpha / \beta$ signaling on muscle-specific transcription? Finally, it will be important to determine how this signaling mechanism influences muscle formation in vivo. Mutating the phosphorylated residue(s) of BAF60 or the residue(s) important for the interaction between BRG1 and MyoD, and targeting these mutants to endogenous loci in mice, could be used to determine the importance of this signaling mechanism in regulating the muscle differentiation program.

1. McKinsey, T.A., Zhang, C.L. \& Olson, E.N. Curr. Opin. Cell Biol. 14, 763-772 (2002).

2. Wu, Z. et al. Mol. Cell. Biol. 20, 3951-3964 (2000).

3. Simone, C. et al. Nat. Genet. 36, 738-743 (2004).

4. Cuenda, A. \& Cohen, P. J. Biol. Chem. 274, 4341-4346 (1999)

5. Zetser, A., Gredinger, E. \& Bengal, E. J. Biol. Chem 274, 5193-5200 (1999).

6. Puri, P.L. et al. Mol. Cell 1, 35-45 (1997).

7. Gerber, A.N., Klesert, T.R., Bergstrom, D.A. \& Tapscott, S.J. Genes Dev. 11, 436-450 (1997).

8. de la Serna, I.L., Carlson, K.A. \& Imbalzano, A.N. Nat. Genet. 27, 187-190 (2001).

9. Molkentin, J.D., Black, B.L., Martin, J.F. \& Olson, E.N. Cel/ 83, 1125-1136 (1995).

10. Zhao, M. et al. Mol. Cell. Biol. 19, 21-30 (1999).

11. Black, B.L., Molkentin, J.D. \& Olson, E.N. Mol. Cell. Biol. 18, 69-77 (1998).

12. Bergstrom, D.A. et al. Mol. Cell 9, 587-600 (2002).

\section{From proteomics to disease}

\section{Kenneth H Kraemer}

The nucleotide excision repair system is essential for repairing DNA damage caused by exposure to sunlight. Now, parallel studies in yeast and individuals with a rare disease called trichothiodystrophy have identified a new component of the DNA repair and basal transcription factor TFIIH.

In 1968, James Cleaver described a defect in DNA repair in cells from three individuals with a rare disease called xeroderma pigmentosum $^{1}$ (Fig. 1). The DNA repair defect in these individuals resulted from a failure of the nucleotide excision repair (NER) system to remove photoproducts from DNA that is damaged by ultraviolet (UV) radiation. Individuals with xeroderma pigmentosum develop pigmentary abnormalities on their skin at an early age and have 1,000 times more sunlight-induced cancers of the skin and eyes ${ }^{2}$. Organisms as diverse as yeast and humans have NER systems composed of homologous proteins that function together to recognize DNA damage, unwind DNA in the damaged region, excise the damage to create a gap and fill in the

Kenneth H. Kraemer is at the Basic Research Laboratory, National Cancer Institute, Bethesda, Maryland 20892-4258, USA. e-mail:kraemerk@nih.gov gap using the undamaged strand as a template (Fig. 1) ${ }^{2,3}$. Defects in seven of these proteins (XPA-XPG) are associated with xeroderma pigmentosum in humans.

In 1993, a group led by Jean-Marc Egly, working to define the basal transcription factor TFIIH, reported that two TFIIH components were also DNA repair proteins: $\mathrm{XPB}$ and $\mathrm{XPD}^{4,5}$. In this issue, Jeffrey Ranish and colleagues $^{6}$ and Giuseppina Giglia-Mari and colleagues ${ }^{7}$ report a new shared component of the DNA repair and basal transcription machinery, called TFB5, which is linked to a rare human disease called trichothidystrophy (TTD).

\section{A crucial link}

The observation of Egly and colleagues established a crucial link between transcription and DNA repair, which explained why mice completely lacking XPB or XPD showed embryonic lethality, as TFIIH is essential for survival. Nevertheless, certain mutations in
ERCC3 (encoding XPB) and ERCC2 (encoding XPD) are present in individuals with xeroderma pigmentosum and are compatible with survival ${ }^{8}$. There are varied clinical phenotypes associated with different defects in XPD and XPB in humans (Fig. 1). Some involve progressive neurologic degeneration, and others involve short stature, developmental delay and severe wasting (the xeroderma pigmentosum and Cockayne syndrome complex). These distinct manifestations seem to reflect defects in different functions of the same protein.

Several years ago, Miria Stefanini and colleagues observed that some individuals with defects in XPD or XPB have a phenotype known as TTD ${ }^{9-11}$. Cells from these individuals behave in culture like those of individuals with xeroderma pigmentosum, but the affected individuals themselves present a very different phenotype characterized by sulfur-deficient brittle hair, skin photosensitivity without increased pigmentation and 